\title{
Genistein modulates gene activity in psoriatic patients
}

\author{
Elwira Smolińska1, Grzegorz Węgrzyn² and Magdalena Gabig-Cimińska1,3四 \\ 1Department of Medical Biology and Genetics, University of Gdańsk, Gdańsk, Poland; 2Department of Molecular and Cellular Biology, \\ Intercollegiate Faculty of Biotechnology UG-MUG, Gdańsk, Poland; 3institute of Biochemistry and Biophysics, Polish Academy of Sciences, \\ Laboratory of Molecular Biology, Gdańsk, Poland
}

\begin{abstract}
Despite the impressive advancements in the treatment of psoriasis over the past two decades, there is still a need for further improvement. As previously shown in the literature, genistein (5,7-dihydroxy-3-(4-hydroxyphenyl)chromen-4-one), naturally occurring plant compound displays multidirectional action, also in relation to alleviating psoriasis symptoms. In this work we focused our attention on genistein impact on expression of genes when treating moderate-to-severe psoriasis patients. Testing the effects of this isoflavone on transcript levels in both skin specimens and peripheral blood cells of four psoriatic subjects, we found that this compound modulated activities of genes coding for anti-psoriatic members and anti-inflammatory mediators of inflammation. It impairs the activity of certain genes which are overexpressed in psoriasis, while stimulating the expression of other transcripts that are repressed in dermatosis.
\end{abstract}

Key words: psoriatic patients; flavonoid genistein; skin biopsy; peripheral blood cells; inflammation

Received: 30 January, 2019; revised: 22 February, 2019; accepted: 23 February, 2019; available on-line: 28 February, 2019

Ue-mail: m.gabig@biol.ug.edu.pl

Abbreviations: ADA, antidrug antibodies; BSA, Body Surface Area; CCL2, C-C motif chemokine ligand 2; CCL4, C-C motif chemokine ligand 4; CCL5, C-C motif chemokine ligand 5; CEBK, Central Register of Clinical Trials; CXCL10, C-XC motif chemokine ligand 10; dbGAP, database of genes associated with psoriasis; DEFB4, human $\beta$-defensin 2; FC, fold change; FDA, Food and Drug Administration; FOXO1, transcription factor forkhead box protein O1A; GEN, Genistein; GL, Glyteer; IL1B, interleukin 1B; IL6, interleukin 6; IL8, interleukin 8; NF-KB, nuclear factor kappa B; PAGD, psoriasisassociated genes database; PASI, Psoriasis Area and Severity Index; PBCs, perpherial blood cells; PGA, Physician's Global Assessment; $\mathrm{PI}$, peptidase inhibitor 3; PI3K/Akt, phosphoinositide 3 kinase/protein kinase B; PN, psoriatic normal skin; PN_D0, uninvolved psoriatic skin on day 0; PN_D56, uninvolved psoriatic skin on days 56; PP, psoriatic plaque skin; PP_D0, lesional involved psoriatic skin on day 0; PP_D56, lesional involved psoriatic skin on days 56 ; RPLP0, ribosomal protein lateral stalk subunit PO; SERPINB3, serpin family B member 3; TNF-a, tumor necrosis factor $a$; UPL, Universal Probe Library; YWHAZ, tyrosine 3-monooxygenase/ tryptophan 5-monooxygenase activation protein zeta

\section{INTRODUCTION}

Choice of the suitable medical handling depends on the severity of psoriasis. If skin lesions are mild and localised, only topical treatment is applied. At a more advanced stage of psoriasis, systemic therapy is initiated, according to clinical recommendations. Another approach of general treatment is suppressing an excessive immune response by using the selected, classic drugs. Over the past decade, biological drugs have been developed, which are an additional treatment option for moderate and severe psoriasis. Unfortunately, $1 / 3$ of patients do not respond to biological therapy, and some lose the initial response due to the development of antidrug antibodies (ADA) (Hsu et al., 2014) Alternatives are smallmolecule drugs and organic compounds targeting the key molecules of selected signalling pathways. Another group of drugs are the so-called biosimilars, which do not show clinically relevant differences in purity, safety and potency relative to a reference therapeutic product. Three of them obtained approval from the Food and Drug Administration (FDA) in 2016; however, further studies are necessary (Gupta et al., 2014; Lee et al., 2013). Treatments used in psoriasis are still of risk of toxicity and adverse reactions. Thus, alternative, safe, and if only possible, natural therapeutic agents are desired.

Bioactive plant-derived metabolites such as flavonoids, have been studied as potential agents in medicine for many years (Molyneux et al., 2007; Nakamura et al., 2010). They are ubiquitous plant secondary products, well-known for their antioxidant and anti-inflammatory properties (Chamcheu et al., 2017; Nijveldt et al., 2001, Pazyar et al., 2015). Among these compounds, genistein (5,7-dihydroxy-3-(4-hydroxyphenyl) chromen-4-one), the major metabolite of soy, is considered as regulator of inflammatory responses in the skin, and therefore can consequently be useful in the treatment of atopic dermatitis, vitiligo and psoriasis (Korkina et al., 2012). Importantly, genistein is a potent inhibitor of the production of proinflammatory factors, such as IL1, IL6, and TNF-alpha by modulating the nuclear factor kappa $\mathrm{B}(\mathrm{NF}-x \mathrm{~B})$ and phosphoinositide 3 kinase/protein kinase B (PI3K/Akt) pathways in macrophages and endothelial cells. Defining molecular bases of genistein biological action and function in psoriasis is essential to evaluate safety of this composite and to develop finest therapeutic procedures. Therefore, in this study we aimed at determining the pattern of isoflavone genistein impact of on genes' expression in the clinical material, skin specimens and blood samples, derived from psoriatic patients.

\section{MATERIALS AND METHODS}

Ethical agreement and patient profile. This multicenter, randomized clinical study was conducted in Poland. Institutional review boards at each study site approved the protocol, and all tested patients provided written informed consent. The investigation was conducted in compliance with the Declaration of Helsinki. The study no. GE/BF/09/13 was registered in the Central Register of Clinical Trials (CEBK) and Independent Ethic Committee under the numbers UR.DBL. BLE.475.0110.2014 and KB/910/13, respectively. Patients were eligible for study enrolment if they were aged 18 years or older with active, clinically stable mild to 
Table 1. Clinical trial design.

\begin{tabular}{|c|c|c|c|c|c|}
\hline & & \multicolumn{4}{|c|}{ Groups identified for study } \\
\hline & & \multicolumn{4}{|c|}{ Trancription analysis } \\
\hline & & Placebo & $75 \mathrm{mg}$ GEN & & $150 \mathrm{mg} \mathrm{GEN}$ \\
\hline & & $n=1$ & $n=2$ & & $n=1$ \\
\hline \multicolumn{2}{|l|}{ Patient } & u. 11 & u.09 & u.12 & u.15 \\
\hline \multicolumn{2}{|l|}{ Age } & 34 & 51 & 60 & 39 \\
\hline \multicolumn{2}{|l|}{ Sex } & male & male & female & female \\
\hline \multicolumn{2}{|l|}{ Race } & caucasian & caucasian & caucasian & caucasian \\
\hline \multicolumn{2}{|l|}{ Weight $(\mathrm{kg})$} & 94 & 98 & 67 & 80 \\
\hline \multicolumn{2}{|l|}{ BMI } & 29.7 & 33.5 & 26.5 & 29.4 \\
\hline \multirow{2}{*}{ PASI } & Day 0 & 7.0 & 3.6 & 11.7 & 9.2 \\
\hline & Day 56 & 8.4 & 8.8 & 5.2 & 16.8 \\
\hline \multirow{2}{*}{ BSA } & Day 0 & 3.0 & 1.5 & 9.75 & 7.5 \\
\hline & Day 56 & 8.5 & 2.5 & 9.0 & 10.5 \\
\hline \multirow{2}{*}{ PGA } & Day 0 & 2 & 2 & 2 & 2 \\
\hline & Day 56 & 2 & 3 & 2 & 3 \\
\hline
\end{tabular}

moderate chronic plaque psoriasis for $\geq 12$ months prior to the first dose of the study drug. Patients were required to have a Psoriasis Area and Severity Index (PASI) score $\leq 12$, a Physician's Global Assessment (PGA) score of 2 or 3, and $<10 \%$ Body Surface Area (BSA) psoriasis involvement (Table 1). Patients were required to have adequate haematological, renal, and hepatic function.

Genistein dosing, skin biopsies and blood sampling. Genistein dosing and blood and skin biopsy preparation for gene profile evaluation: Patients were screened 1-21 days prior to the administration of the first dose of genistein or placebo on day 0 . They were enrolled in this multicenter, double-blind, placebo-controlled study, randomized $(1: 1)$ to treat with oral filmcoated tablets in one of two treatment regimens: (1) genistein $75 \mathrm{mg}$ or $150 \mathrm{mg}$ per day or (2) placebo. Genistein (GEN) was synthesised at the Pharmaceutical Research Institute (Warsaw, Poland) (Table 1).

Samples of blood and skin biopsy were collected at two time points: baseline (Day 0) and 56 days (Day 56) of treatment. Biopsies were taken under local anaesthesia from 4 psoriatic participants. One $3 \mathrm{~mm}$ punch biopsy was obtained from psoriatic plaque skin (PP) and the other psoriatic normal skin (PN), sampled at least $10 \mathrm{~cm}$ away from any active plaque. Venous blood for gene expression profiling was collected by phlebotomy with a butterfly needle into PAXgene tubes. The first drawn tube from all patients was ejected, as suggested by the PAXgene procedure.

\section{RNA processing}

Skin biopsies. After removal from the skin, biopsies were snap-frozen in liquid nitrogen and stored at $-80^{\circ} \mathrm{C}$ until use. The isolation of total RNA from psoriatic plaque and psoriatic normal skin (unaffected skin) biopsies was carried out using an RNA Fibrous Tissues Mini Kit (Qiagen, Germany) and Trizol (Ambion, USA), according to the manufacturer's protocol. Blood. Total RNA was extracted from the whole blood of patients and control subjects using the PAXgene Blood RNA Kit (Qiagen, Germany) according to the manufacturer's protocol. Analysis of RNA quality and quantity was performed using the Nanodrop (ThermoFisher Scientific,
USA) and RNA 6000 Nano Assay on the Agilent 2100 Bioanalyser (Agilent Technologies, USA).

\section{Real-time quantitative reverse transcription PCR (real- time qRT-PCR)}

The reverse transcription reaction was performed on an RNA template and cDNA was synthesised using the Transcriptor First-Strand cDNA Synthesis Kit (Roche Applied Science, USA) according to the manufacturer's protocols. All probes for real-time qRT-PCR are summarised in Table 2. The 63 genes were selected on the basis of the classification criteria referring to the most up-to-date scientific reports describing a list of genes with significantly differentiated activity obtained in both in vitro and in vivo analyses when studying psoriatic phenotypes, stated at least a couple of times in unrelated literature sources (Bocheńska et al., 2017; Coda et al., 2012; Emmerson et al., 2010; Galluzzo et al., 2018; González et al., 2011; Gudjonsson et al., 2009; Gudjonsson et al., 2010; Martin et al., 2013; Tian et al., 2012). Except for published lists of genes, we also studied and reviewed the psoriasis-associated genes database (PAGD), as well as the database of genes associated with psoriasis (dbGAP). Thus, applied real-time qRT-PCR assays were obtained for genes encoding proteins involved in lipid metabolism, antimicrobial defences, epidermal differentiation and the control of cutaneous vasculature. As keratinocytes at inflammatory sites of the epidermis induce the abnormal expression of cytokines and chemokines, leading to the infiltration of $\mathrm{T}$ cells or leukocytes (Tian et al., 2012; Dowlatshahi et al., 2013), we also examined the effects of genistein on the expression of selected chemokine genes.

The pre-plated PCR assays contained target specific primers and a matching probe from the Universal Probe Library (UPL). Real-time qRT-PCR was run using the Light Cycler 480 II detection system (Roche Applied Science, USA) and the quantification of gene expression was determined by the comparative $\Delta \Delta \mathrm{Ct}$ method. The analysis of transcripts driven from the tested genes showed those in which expression was identified as being different by at least 0.7 - and 1.3-fold changes $(p<0.05)$ : (skin samples) in lesional involved psoriatic 
Table 2. Sequences of primer and probe numbers applied for real-time qRT-PCR.

\begin{tabular}{|c|c|c|c|c|c|}
\hline Gene symbol & Forward sequence & Reverse sequence & UPL probe no. & Skin & PBCs \\
\hline ACACB & GGGAGAGAACCGGGAAGA & CCTCGGATGGACAGTTCCT & 77 & $\neq$ & \\
\hline AKT1 & GCAGCACGTGTACGAGAAGA & GGTGTCAGTCTCCGACGTG & 45 & & $\neq$ \\
\hline CAT & CGCAGTTCGGTTCTCCAC & GGGTCCCGAACTGTGTCA & 67 & $\neq$ & \\
\hline CCL2 & AGTCTCTGCCGCCCTTCT & GTGACTGGGGCATTGATTG & 83 & & $\neq$ \\
\hline CCL3 & GCTGACTACTTTGAGACGAGCA & GCTTCGCTTGGTTAGGAAGA & 125 & & $\neq$ \\
\hline CCL4 & TGCTTCTCCATGAGACACATCT & AAAGATTAAATTAAGAGAAGGGACAGG & 55 & $\neq$ & $\neq$ \\
\hline CCL5 & ССTCATTGCTACTGCCCTCT & GGTGTGGTGTCCGAGGAATA & 16 & $\neq$ & $\neq$ \\
\hline CCL19 & CACTACCTTCTCATCAAGGATGG & CCCCTCAGTGTGGTGAACA & 40 & & $\neq$ \\
\hline CXCL1 & CATCGAAAAGATGCTGAACAGT & CTTCAGGAACAGCCACCAGT & 52 & $\neq$ & $\neq$ \\
\hline CXCL6 & CACGAGGAAACCAAAGTGC & GGGAGGCTCATAGTGGTCAA & 65 & & $\neq$ \\
\hline CXCL10 & AAAAGGTATGCAATCAAATCTGC & AAGAATTTGGGCCCCTTG & 86 & $\neq$ & $\neq$ \\
\hline DEFB4A & TCAGCCATGAGGGTCTTGTA & GGATCGCCTATACCACCAAA & 35 & $\neq$ & $\neq$ \\
\hline EGFR & CAGCCACCCATATGTACCATC & AACTITGGGCGACTATCTGC & 42 & & $\neq$ \\
\hline FABP5 & GCAGACCCCTCTCTGCAC & TCGCAAAGCTATTCCCACTC & 11 & $\neq$ & \\
\hline FADS1 & CATCAACATGCATCCCTTCTT & TITGTGCTGGTGGTTGTACG & 60 & $\neq$ & \\
\hline FOSL1 & AGGAACTGACCGACTTCCTG & TCTGCAGCTCCTCAATCTCTC & 26 & $\neq$ & \\
\hline FOXC1 & TGCTTITCAGAGACCTGCTIT & GCAAGGAAGAAGGCAAGAGA & 52 & $\neq$ & \\
\hline FOXO1 & GCAGCCAGGCATCTCATAAC & GCATGTCCAGGGTGGGTAT & 33 & $\neq$ & \\
\hline GATA3 & CTCATTAAGCCCAAGCGAAG & GTCTGACAGTTCGCACAGGA & 108 & & $\neq$ \\
\hline GJB2 & CAAGAACGTGTGCTACGATCA & GCGTGGACACGAAGATCAG & 60 & $\neq$ & \\
\hline IL1A & GAATGACGCCCTCAATCAAA & TGATGACTTATAAGCACCCATGTC & 66 & $\neq$ & $\neq$ \\
\hline IL1B & AGCTGATGGCCCTAAACAGA & TCGGAGATTCGTAGCTGGAT & 85 & $\neq$ & $\neq$ \\
\hline IL1RN & TGCCTGTCCTGTGTCAAGTC & TCTCGCTCAGGTCAGTGATG & 54 & $\neq$ & $\neq$ \\
\hline IL2 & TGTTCAAGAGTTCCCTATCACTCTC & GCAAGACAGGAGTTGCATCC & 51 & & $\neq$ \\
\hline IL6 & ACCGGGAACGAAAGAGAAG & GAAGGCAACTGGACCGAAG & 133 & $\neq$ & $\neq$ \\
\hline IL8 & TAGCCAGGATCCACAAGTCC & CTGTGAGGTAAGATGGTGGCTA & 98 & $\neq$ & $\neq$ \\
\hline IL10 & CTGCCTAACATGCTTCGAGA & GTCCAGCTGATCCTTCATTTG & 161 & $\neq$ & $\neq$ \\
\hline IL11 & AGCTGCAAGGTCAAGATGGT & AGATTGTTTCCAGTTTGCTATGG & 76 & & $\neq$ \\
\hline IL12A & CACTCCCAAAACCTGCTGAG & TCTCTTCAGAAGTGCAAGGGTA & 50 & $\neq$ & $\neq$ \\
\hline IL17A & CCTATAAGCAAAACAAAGCATGTC & CGAGAACTTGGAATTITGGGTA & 85 & & $\neq$ \\
\hline IL18 & AAGGAATTGTCTCCCAGTGC & CCTCTTCCCGAAGCTGTGTA & 71 & $\neq$ & $\neq$ \\
\hline IL20 & ACAGCCAGATTCTGAGTCACTTT & AGCAGCATCACTTTCCTCCT & 25 & $\neq$ & $\neq$ \\
\hline IL20RA & GCGTACACGTGGAGTCCTTC & AGCCTTGAACTCTGATGATTGAT & 81 & & $\neq$ \\
\hline IL23A & GAAGCTGCTAGGATCGGATATTT & AGTAGGGAGGCATGAAGCTG & 32 & $\neq$ & $\neq$ \\
\hline IL24 & GCTGCAGCAGGAGGTTCT & GCAGGGTGTGGACAAGGTAA & 89 & & $\neq$ \\
\hline JAK1 & AATGGCTGTCATGGTCCAAT & TACATCCCCTCCTCGCTTC & 147 & $\neq$ & $\neq$ \\
\hline JAK2 & CAGGAACAAGATGTGAACTGTTIC & CCCATGCAGAGTCTIITCAG & 52 & $\neq$ & $\neq$ \\
\hline JUN & AGGATAGTGCGATGTTTCAGG & GACTTCTCAGTGGGCTGTCC & 65 & & $\neq$ \\
\hline KRT6B & GGCCCTGGATGTGGAGAT & TGACTTGTCCAACGCCTTC & 67 & $\neq$ & \\
\hline MMP10 & CAAAAGAGGAGGACTCCAACA & TTCACATCCTTTTCGAGGTTG & 61 & & $\neq$ \\
\hline NFKB1 & CTGGCAGCTCTTCTCAAAGC & TCCAGGTCATAGAGAGGCTCA & 68 & $\neq$ & $\neq$ \\
\hline NFKBIZ & TTGATACCATTAAGTGCCTAATTCA & TTCTTCAGCTGCCAAATGC & 32 & & $\neq$ \\
\hline $\mathrm{NOTCH} 1$ & TCAATGAGTTCCAGTGCGAGT & GCACACTCGTCCACATCGTA & 105 & & $\neq$ \\
\hline PCYOX1 & GCTCCGCCAGATAAAATCG & CAAATTTCTGCCGCAGGT & 66 & $\neq$ & \\
\hline
\end{tabular}




\begin{tabular}{|c|c|c|c|c|c|}
\hline $\mathrm{PI} 3$ & AGCCAGTCAAAGGTCCAGTC & GCGCACCGGATCAAGATA & 51 & $\neq$ & $\neq$ \\
\hline $\mathrm{RHOB}$ & CCCCTGAGCATGCTITCT & CGAGGGGAGTCGAACAGAC & 5 & $\neq$ & \\
\hline RPLPO & TCGACAATGGCAGCATCTAC & GCCAATCTGCAGACAGACAC & 6 & $\neq$ & $\neq$ \\
\hline SERPINB3 & CAACAAGCTCTTCGGAGAAAA & TGGAGCATTTGCAAAATCAA & 147 & $\neq$ & \\
\hline SIGIRR & CTGGTGACCTTGCTGCTCT & TGCACTTCTTTCCAAAAATCG & 12 & $\neq$ & $\neq$ \\
\hline SOCS1 & GGTCCCCCTGGTTGTTGTA & GTAGGAGGTGCGAGTTCAGG & 36 & $\neq$ & $\neq$ \\
\hline SOCS2 & AGGCCTCACTGCAATTTGAT & TGCAAAATATAAAATGCCCAAG & 40 & & $\neq$ \\
\hline SP1 & CTATAGCAAATGCCCCAGGT & TCCACCTGCTGTGTCATCAT & 131 & $\neq$ & $\neq$ \\
\hline STAT1 & GGATTGAAAGCATCCTAGAACTCA & GATGAAGCCCATGATGCAC & 32 & & $\neq$ \\
\hline STAT3 & TCCTGAAGCTGACCCAGGTA & GGTCGTTGGTGTCACACAGAT & 43 & $\neq$ & $\neq$ \\
\hline STAT4 & ATCTGCCTCTATGGCCTGAC & AATCATCACCACAGGCAATG & 54 & & $\neq$ \\
\hline SYNCRIP & CGATACCATCGGACAGGATT & TCTGTAGCCATGTITCCAGAGAT & 30 & $\neq$ & \\
\hline TLR1 & AGGGGACAATCCATTCCAA & TTGGTCTATATTTTTGACAAATTCTCC & 100 & $\neq$ & $\neq$ \\
\hline TLR4 & CAAGATGCCCCTTCCATTT & TCCTTAGGAATTAGCCACTAGACTTT & 94 & & $\neq$ \\
\hline TNF & CGGTGCTTGTTCCTCAGC & GCCAGAGGGCTGATTAGAGA & 12 & & $\neq$ \\
\hline TUFT1 & GGAAAGTCCGGCAAATGATA & GCGTCCTITGACTGGATCAC & 50 & $\neq$ & \\
\hline UST & TGTGGACAGCATCCCAGAT & TCATTCACGTTCAGCTITGC & 116 & $\neq$ & \\
\hline YWHAZ & GATCCCCAATGCTTCACAAG & TGCTTGTTGTGACTGATCGAC & 102 & $\neq$ & $\neq$ \\
\hline ZNF12 & GATCTGCCTCCGCTTTCAC & ACAGCCACGTCCTTGAATG & 65 & $\neq$ & \\
\hline ZNF483 & ACTGGAAAACCTCAGGAACCTA & CATGGCAATTCAACCCACT & 33 & $\neq$ & \\
\hline
\end{tabular}

‡points the analysis of a given gene in the skin or the $\mathrm{PBC}^{\prime}$ samples.

skin (PP_D0) vs. uninvolved psoriatic skin (PN_D0) of u.09, 11, 12 and 15 patients on day 0 (baseline). Also, lesions involved the psoriatic skin of patients following 56 days (PP_D56) of $75 \mathrm{mg}$ (u.09 and 12 patients) or $150 \mathrm{mg}$ (u.15 patient) genistein treatment handling or placebo (u.11 patient) vs. baseline (PP_D0); (blood samples) in u.09, 11, 12 and 15 patient's PBCs vs. normal healthy people (control group) on day 0 (baseline), and in PBCs of patients following 56 days of $75 \mathrm{mg}$ (u.09 and 12 patients) or $150 \mathrm{mg}$ (u.15 patient) genistein treatment handling or placebo (u.11 patient) vs. baseline. Results were normalised to the expression of housekeeping genes such as RPLPO (ribosomal protein lateral stalk subunit P0) and YWHAZ (tyrosine 3-monooxygenase/ tryptophan 5-monooxygenase activation protein zeta).

\section{RESULTS}

\section{Alterations in gene expression in lesional psoriatic skin}

In summary, we have analysed the activity of the 41 genes indicated in Table 2. Due to the limited amount of isolated RNA from patient samples, studies on $n=1$ were performed. In general, in the 4 paired PP (lesional involved psoriatic skin (PP_D0) ) and PN (uninvolved psoriatic skin (PN_D0)) skin sections from 4 patients (u.09, 11,12 and 15) on day 0 (baseline), a rather clear separation of the PN_D0 samples from the PP_D0 samples was revealed (Table 3). On the basis of our criteria for differentially regulated transcripts, we identified that all 41 genes differed in expression between the PP_D0 and PN_D0 samples, at least for one reference (RPLPO or $Y W H A Z$ ) and minimum for the individual patient from the 4 subjects, analysed by real-time qRT-PCR (Table 3). Most of these genes (21 in total) were either down- or up-regulated, or unchanged, or had no data available, depending on the patient being analysed. Another 18 mRNAs revealed up-regulated or unchanged records, or no data accessible, again depending on the subject being studied. Similarly, the activity of two other genes, namely FADS1 and TUFT1, was decreased or remained unaffected depending on the patient being tested. Seven of the 41 modulated genes, visible among all four subjects, gained an increase in expression of as much as 10 to 20 times. These included genes coding for the $\mathrm{C}-\mathrm{C}$ motif chemokine ligand 4 (CCLA), anti-microbial peptides, including human $\beta$-defensin 2 (DEFB4), and transcription factor forkhead box protein O1A (FOXO1), interleukin (IL) family members, such as interleukin 1B (IL1B) and 8 (ILS), peptidase inhibitor 3 (PI3) and serpin family B member 3 (SERPINB3).

\section{Genistein-responsive genes in skin biopsies}

To investigate the impact of genistein on the gene activity profile of inflamed skin (PP), we compared the gene expression of skin biopsy samples at baseline $\left(\mathrm{PP}_{-}\right.$ D0) and after 56 days (PP_D56) of therapy in the four patients, i.e. placebo, 75 or $150 \mathrm{mg}$ genistein-treated. Again, as in the previous experiment, we also analysed the expression of 41 genes (Table 2), with $n=1$ only due to the restricted access to a larger quantity of material, with respect to reference genes, RPLPO and YWHAZ at a constant expression level, by using real-time qRTPCR. Response to genistein was associated with a significant expression change in all 41 of the studied genes, irrespective of the patient, and for at least one reference (Table 3). As in the case of transcripts that differed in level between the PP_D0 and PN_D0 samples, a differentiated profile of gene activity modulation was also observed, depending on the examined patient. In gen- 


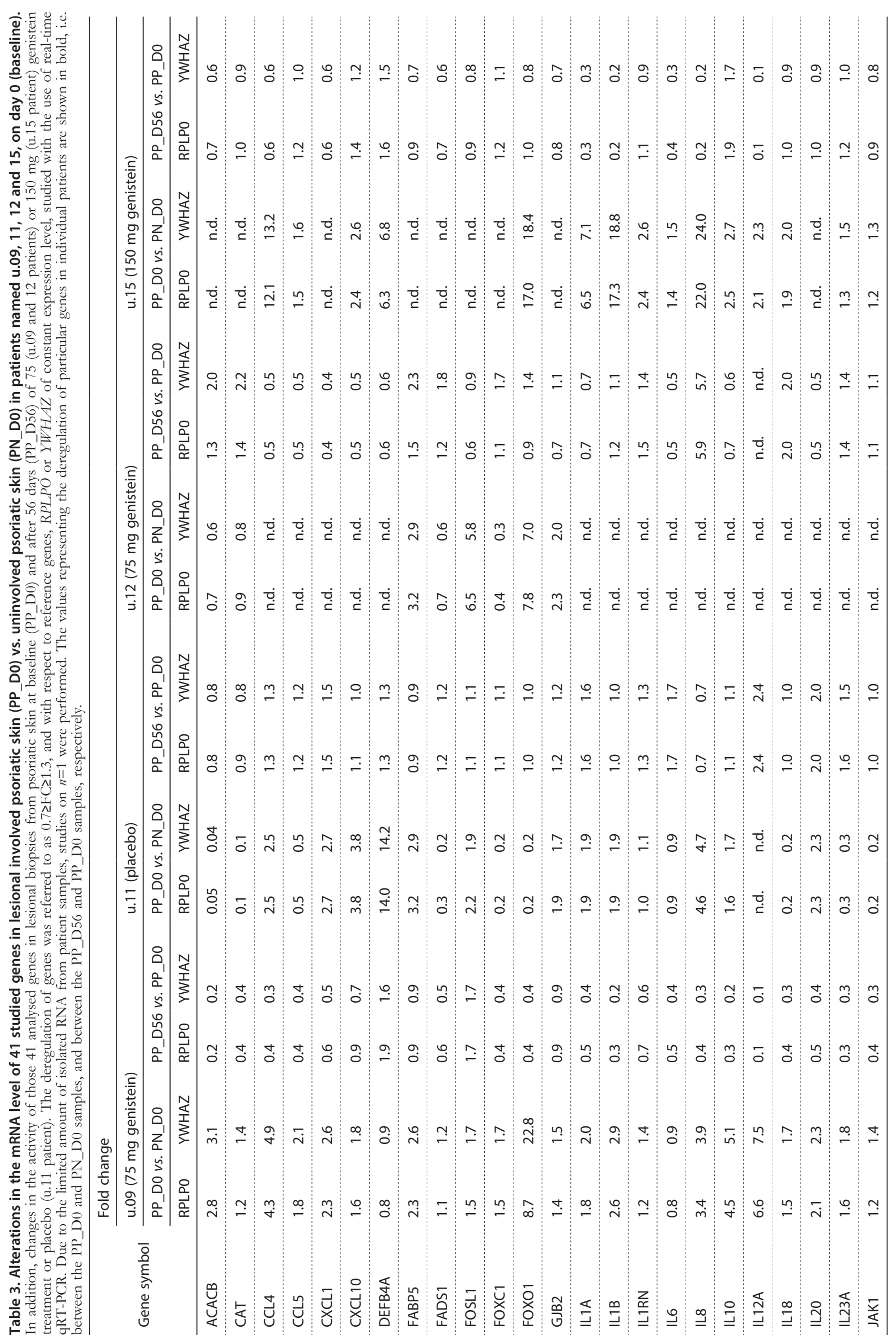






eral, the smallest number of deregulated genes was identified for placebo (u.11). There were 14 genes in total, 12 with increased and 2 with decreased expression. Studying carefully the gene expression levels modulated in placebo, we found that stimulated CCL4, CXCL10, IL1A and IL6 were down-regulated for all three patients treated with genistein. The other ten genes with modulated activity in placebo exhibited a substantially varied pattern of expression in individual patients treated with genistein. In turn, the highest number of deregulated genes was observed for patient u.09, where all of the studied mRNAs, except for FABP5 and GJB2, revealed a change in the level at PP_D56 vs. PP_D0. Furthermore, in the case of both doses of 75 (u.09) and 150 (u.15) $\mathrm{mg}$ genisteintreated patients (PP_D56 vs. PP_D0), most of the genes were down-regulated and only few showed accelerated activity once exposed to the tested isoflavone. However, the opposite trend was found for patient 12; that is, with a significant majority of up-regulated genes. In addition, the most significantly deregulated among all three patients in response to genistein (PP_D56 vs. PP_D0) was IL8, depending on the subject, from 3 to 5 times, exhibiting either a decrease in mRNA level (for u.09 and 15, respectively) or an increase (around 6 times) in transcript amount (for u.12).

It should be noted that all 14 of the deregulated genes in u.11 placebo were found among the 38 transcripts (while for IL12A transcript we had no data available) which differed in level between the PP_D0 and PN_D0 samples (Table 3). Likewise, for genistein-treated patients, we identified a number of genes in a set of the transcripts that differed in levels between the PP_D0 and PN_D0 samples. Correspondingly, we found that most of the genes (except for DEFB4A, FOSL1, KRT6B and PI3 in case of u.09; and SIGIRR, SP1, TLR1, TUFT1 and UST in case of u.15), being deregulated in PP vs. PN on day 0 , showed a considerable reduction in activity after genistein handling (D56). For u.12, we found 11 of the 18 genes with modulated activity in the PP_D0 vs. PN_D0 were stimulated in response to genistein (PP_D56 vs. PP_D0).

\section{Changes in gene activity in the PBCs of psoriatic patients}

To identify genes that were differentially regulated in the PBCs of u.09, 11, 12 and 15 psoriatic patients, we examined the expression profile of the 45 genes listed in Table 2. In total, 16 out of 45 transcripts that differed in level between the psoriatic patients' and were varied from normal healthy control PBCs, in respect to RPL$P O$ reference, and minimum for the individual patient from the 4 subjects, analysed 
Table 4. List of 16 deregulated genes of the 45 studied in psoriatic PBCs, i.e. genes differentially expressed between u.09, 11,12 and 15 psoriatic patients and the normal healthy people on day 0 (baseline), analysed via real-time qRT-PCR.

Alterations in transcript amount of the selected genes relate to an expression ratio greater than or equal to 1.3-fold and below or equal to 0.7 , and with respect to RPLPO mRNA expression at a constant level. Due to the limited amount of isolated RNA from patient samples, studies on $n=1$ were performed.

\begin{tabular}{|c|c|c|c|c|}
\hline \multirow{2}{*}{ Gene symbol } & \multicolumn{4}{|l|}{ Fold change } \\
\hline & u.09 (75 mg genistein) & u.11 (placebo) & u.12 (75 mg genistein) & u.15 (150 mg genistein) \\
\hline CCL2 & 0.5 & 0.5 & 0.2 & 0.8 \\
\hline CCL5 & 0.6 & 0.7 & 0.7 & 0.9 \\
\hline CXCL6 & 1.6 & 2.6 & 2.6 & n.d. \\
\hline CXCL10 & 1.8 & 2.2 & 3.5 & 4.6 \\
\hline IL $1 A$ & 0.9 & 1.9 & 0.9 & 1.3 \\
\hline $\mathrm{IL} 2$ & 2.7 & n.d. & 4.4 & n.d. \\
\hline IL6 & 0.6 & 1.2 & 1.8 & 2.4 \\
\hline IL8 & 2.6 & 1.4 & 1.7 & 6.1 \\
\hline IL10 & 0.6 & 1.1 & 0.1 & 0.5 \\
\hline IL11 & 1.0 & 4.6 & 6.2 & 8.7 \\
\hline IL12A & 0.4 & 0.4 & 0.2 & 0.9 \\
\hline IL17A & 0.4 & 1.7 & 4.3 & n.d. \\
\hline JUN & 1.0 & 1.8 & 2.4 & 5.4 \\
\hline SOCS2 & 0.6 & 0.7 & 0.8 & 0.8 \\
\hline STAT4 & 0.7 & 0.5 & 0.7 & 1.0 \\
\hline TLR1 & 0.9 & 1.5 & 0.7 & 2.6 \\
\hline
\end{tabular}

n.d. shortcut stands for no data available.

by real-time qRT-PCR were affected (Table 4). Due to the limited amount of isolated RNA from patient samples, studies of $n=1$ were performed. In common between the four psoriatic patients when studying their PBC expression profiles, two genes revealed increased activity (i.e. CXCL10 and IL8, coding for the C-X-C motif chemokine ligand 10 and interleukin 8, respectively), while another two were down-regulated (CCL2 and CCL5, correspondingly coding for the $\mathrm{C}-\mathrm{C}$ motif chemokine ligand 2 and $\mathrm{C}-\mathrm{C}$ motif chemokine ligand 5 , respectively) (Table 4). The largest number of genes with modulated activity (14 genes) was observed for patient u.12 (treated with $75 \mathrm{mg}$ genistein). Among them 8 genes were up-regulated, as is the case for subject u.11. In turn, the smallest number of genes (i.e. 8) with the only single down-regulated gene had u.15 subject (taking $150 \mathrm{mg}$ genistein).

\section{Modulated transcripts' level in the PBCs in response to genistein}

To study the effect of genistein on the gene expression profile of PBCs from psoriatic subjects treated with $75 \mathrm{mg}$ (u.09 and 12 patients) and $150 \mathrm{mg}$ (u.15 patient) genistein or placebo (u.11 patient), we compared the mRNA levels at baseline (day 0) and after 56 days of therapy with respect to housekeeping RPLPO and $Y W$ $H A Z$ genes and by real-time qRT-PCR (Fig. 1). Once more, as in the previous experiment, we also analysed the expression of 45 genes in total (Table 2), with $n=$ 1 only due to the restricted access to a larger quantity of material. The analysis revealed significant alterations in the activity of 36 of the 45 genes (Fig. 1). Then, we realised that there were 15 transcripts among those 36 genes (except for SOCS2) that differed in levels between the psoriatic patient PBCs and those derived from normal healthy controls.

Wherein the largest number of genes (i.e. 23) with modulated activity was for u.11 (with 21 up-regulated and 2 down-regulated), the fewest genes (i.e. 7) were reported for patient u.15 (Fig. 1). Of the 36 genes with modulated activity, only two, CCL2 and IL10, were deregulated, albeit diversified, in all 4 subjects tested. The most significantly stimulated were genes in patients treated with $75 \mathrm{mg}$ genistein (i.e., IL17 $A$ app. 6-fold for u.09 and IL10 app. 8-fold for u.12). On the other hand, the most considerably, as much as 3 to 6 times, down-regulated were IL6, IL11, IL17A and CXCL6 genes, with patient u.12 taking $150 \mathrm{mg}$ genistein.

It is worth adding that, as in the case of gene activity analyses in skin specimens, where it was possible to determine a set of common genes between those with modulated activity in patients entering genistein tests and those that differed in level between the PP_D0 and PN_D0 samples, a similar circumstance was observed in PBC studies. Thus, 7 of the 23 genes with modulated activity in u.11 placebo (Fig. 1) were found among the 11 transcripts that differed in levels between the psoriatic patients' and derived from normal healthy control PBCs (Table 4). Similarly, for genistein-treated patients we identified a number of genes in a set of the transcripts that differed in level between the PBC of psoriatic patients and normal healthy controls. Respectively, we found 7 of the 16 genes to have modulated activity in the case of u.09 (Fig. 1) from 11 transcripts (Table 4), 11 of the 20 genes with modulated activity in the case of u.12 (Fig. 1) from 14 transcripts (Table 4), and 2 of the 7 genes with modulated activity present in u.15 (Fig. 1) from 8 transcripts (Table 4). Remarkably, our results showed that the aberrant expression of some of these 


\section{u.09 patient}

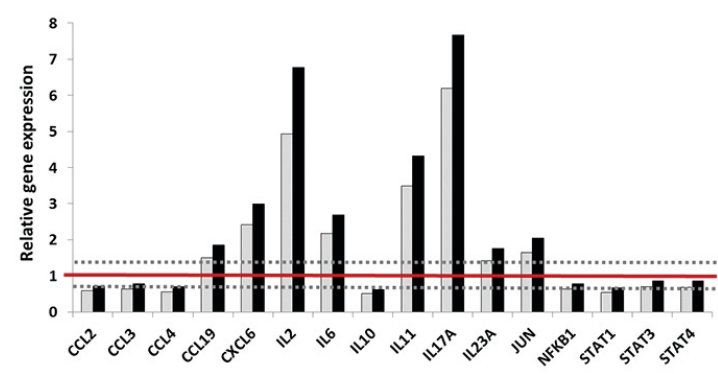

u.11 patient

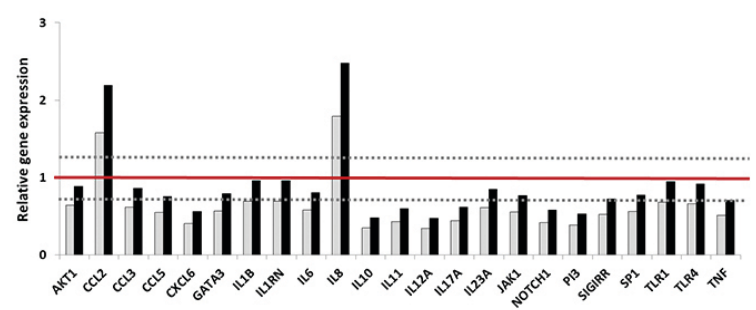

\section{u.12 patient}

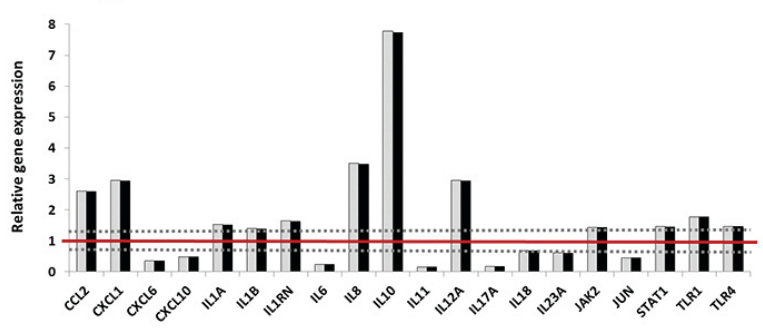

u.15 patient

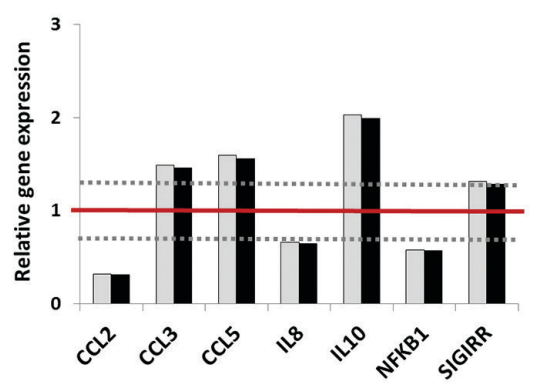

Figure 1. Alterations in the activity of 36 genes in PBCs from psoriatic subjects:

treated with $75 \mathrm{mg}$ (u.09 and 12 patients) and $150 \mathrm{mg}$ (u.15 patient) genistein or placebo (u.11 patient) on Day 56 vs. Day 0 (baseline, red line), analysed with the use of real-time GRT-PCR are referred to as $0.7 \geq F C \geq 1.3$ (grey lines). The results are shown relative to the expression of the housekeeping genes RPLPO (grey bars) and YWHAZ (black bars). Due to the limited amount of isolated RNA from patient samples, studies on $\mathrm{n}=1$ were performed.

genes affected in psoriasis (Table 4) are improved by the action of genistein (Fig. 1).

\section{Deregulated profile of gene expression in the skin specimens and the PBCs in response to genistein}

Also noteworthy is the comparison of gene activity in the skin specimens and PBCs of u.09, 11, 12 and 15 patients participating in the genistein test (Fig. 2). Because the PBC analysis was basically representative of inflammation-immune axis regulation genes, this comparison is just about these mRNAs. It was possible to produce a refined list of genes that were consistently differentially expressed in both PP skin and PBCs derived from all three patients treated with genistein, while not for placebo. Within this list, there are several genes that were deregulated; for patient u. 09 we found 4 down-regulated transcripts (CCL4, IL10, NFKB1 and STAT3), for subject u.12 there were 2 decreased (CXCL10 and ILO) and 1 stimulated in activity (ILIRN), and in the case of patient u.15, there was one with increased (ILS) and one reduced (IL10) mRNA level.

\section{DISCUSSION}

Various topical medications, phototherapy, and systemic drugs are available to treat patients with psoriasis of varying disease severity. However, many studies are constantly performed to detect new therapeutic anti-psoriatic substances, which are verified in preclinical laboratory tests and clinical phase trials (Bocheńska et al., 2017). This includes natural compounds which, as it turns out, are important in the treatment of psoriasis. They are the starting point for further analyses to improve the health and quality of patients' lives. Genistein turn out to be very important here. For example, Ito's group investigated the topical application of Glyteer (GL, soybean) on a psoriatic model in mice (Ito et al., 1992). Also, topical genistein was found to decrease psoralen-ultraviolet A (PUVA)-induced skin thickening and greatly reduce cutaneous erythema and ulceration in a dose-dependent manner (Shyong et al., 2002). Genistein, a soy-derived molecule has attracted attention as a potent agent in the treatment of psoriasis, not only due to its anti-proliferative and immunosuppressive properties, but also as a mediator modulating the expression of various genes, whose products are involved among others in different phases of inflammation and proliferation (Smolińska et al., 2018). These statements resulted in proposing the performance of test with the use of genistein for monitoring the activity of genes in the material derived from psoriatic patients.

Our study was designed to evaluate the impact of two dosing regimens of genistein at 75 or $150 \mathrm{mg}$ per day administered orally on gene expression in patients with active mild to moderate chronic plaque psoriasis.

The exploratory objectives of this study were to evaluate gene expression levels in the four patients, i.e. placebo (u.11), 75 (u.09 and 12) or 150 (u.15) mg genistein-treated. In total, with the criteria referred to in the Methods section, we selected 64 genes coding for proteins involved in lipid metabolism, antimicrobial defences, epidermal differentiation and control of cutaneous vasculature, as well as immune mediators, for the analyses of 41 transcripts in the skin and $45 \mathrm{mRNAs}$ in PBC samples, correspondingly (Table 2). Indeed, it has been shown in the literature that genistein influences the expression of inflammatory mediators, including the down-regulation of cytokine genes involved in mediating communication between cells (Mirahmadi et al., 2018; 
u.09 patient

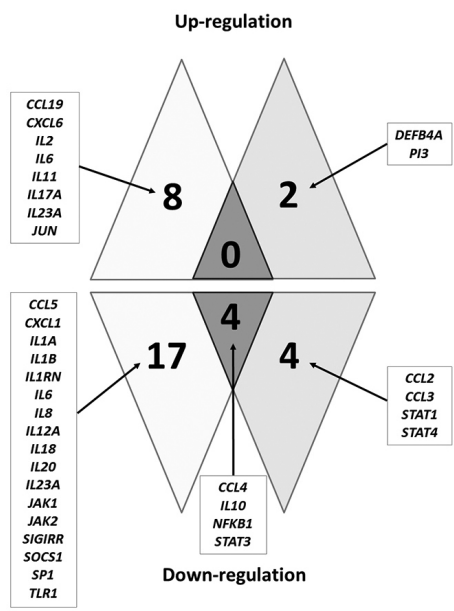

u.11 patient



u.12 patient

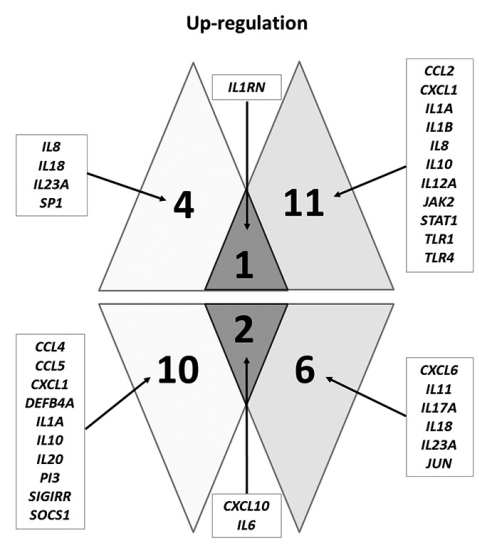

Down-regulation

u.15 patient

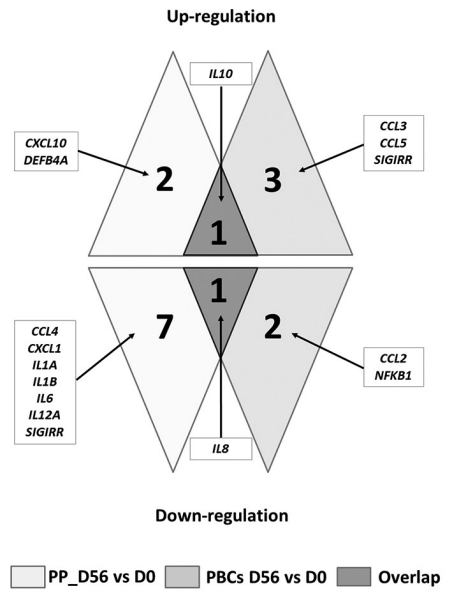

Figure 2. Genes identified as changed under studied conditions with corresponding overlap between the datasets $(0.7 \geq F C \geq 1.3)$. Up- and down-regulated genes of the 64 analysed transcripts in lesional involved psoriatic skin (PP) and in peripheral blood cells (PBCs) of patients $\mathrm{u} .09,11,12$ and 15 on day 0 (D0), and following 56 days (D56) of genistein treatment or placebo.

Vivatvakin et al., 2017). Initially, however, we dealt with the analysis of gene activity in those four patients before genistein administration. The study was in lesional involved psoriatic skin (PP_D0) vs. PN (uninvolved psoriatic skin (PN_D0), and in the PBCs derived from psoriatic patients $v$ s. the normal healthy people. The results of these investigations revealed the clear separation of both the PN_D0 samples from the PP_D0 samples, as well as psoriatic PBC samples from the PBCs of normal healthy subjects (Table 3 and 4). In the next step, we compared the gene expression in the same four patients, either treated with 75 or $150 \mathrm{mg}$ genistein, in relation to untreated placebo, again in both of the skin biopsy samples at baseline (PP_D0) and at 56 days (PP_D56) of therapy, and for the PBC samples at day 0 and 56. The outcomes of these works, described in detail in the Results section, allowed significant alterations in gene activity in both skin biopsies and PBCs under the influence of genistein to be noted (Table 3 and Fig. 1). It impairs the activity of certain genes which are overexpressed in psoriasis, while stimulating the expression of other transcripts which are repressed in this dermatosis. Furthermore, the tissue-cross consideration regard- ing the activity assessment of inflammation-immune axis genes in the material of the skin specimens and PBCs of our four patients joining the genistein test allow us to conclude that this isoflavone exhibits potential as an active anti-inflammatory agent, associated with its capacity to modify the pattern of transcript levels across skin and blood cells (Fig. 2). Also, we found genes that were consistently differentially expressed both in PP skin and PBCs derived from all three patients treated with genistein, while not for placebo.

Moreover, taking into consideration our previous results on the use of "psoriasis-like" HaCaT keratinocytes exposed to isoflavone genistein (Smolińska et al., 2018), we found that the $10 \mathrm{mRNAs}$ (i.e. CCL4, CXCL1, CXCL10, IL1A, IL6, IL8, KRT6B, PI3, SIGIRR and ZNF483) studied in psoriatic skin patients were among the genes which were deregulated in the genistein-treated "psoriasis-like" epithelial cell line $\mathrm{HaCa}$ T. In turn, when analysing in detail the individual patients, we found 28 transcripts deregulated regarding the modulation profile by genistein in patient u.09, 22 in patient u.12 and 14 in patient u.15, as it was in case of "psoriasis-like" keratinocytes (see Figure 3 in Smolińska et al., 2018). 
To the point, we may conclude that, isoflavone genistein, previously seen to be able to modulate the antipsoriatic members and anti-inflammatory mediators of inflammation, in this study showed some benefits to the aberrant expression of genes contributing to the progression of this dermatosis.

\section{Acknowledgements}

This work was supported by the National Centre for Research and Development (project grant no. NCRD PBS1/B7/7/2012), the National Science Centre (UMO$2017 / 27 / \mathrm{B} / \mathrm{NZ3} / 02212$ ), and by the Institute of Biochemistry and Biophysics of the Polish Academy of Sciences (task grant no. T-32.1). The authors thank Biofarm Sp. z o.o. from Poznań and MTZ Clinical Research from Warsaw that have facilitated the undertaking of this study, for their cooperation and support within the project no. NCRD - PBS1/B7/7/2012.

\section{REFERENCES}

Bocheńska K, Smolińska E, Moskot M, Jakóbkiewicz-Banecka J, Gabig-Cimińska M (2007) Models in the research process of psoriasis. Int J Mol Sci 18: 2514. https://doi.org/10.3390/ijms18122514

Chamcheu JC, Adhami VM, Esnault S, Sechi M, Siddiqui IA, Satyshur KA, Syed DN, Dodwad SM, Chaves-Rodriquez MI, Longley BJ (2017) Dual inhibition of PI3K/Akt and mTOR by the dietary antioxidant, delphinidin, ameliorates psoriatic features in vitro and in an imiquimod-induced psoriasis-like disease in mice. Antioxid Redox Signal 26: 49-69. https://doi.org/10.1089/ars.2016.6769

Coda AB, Icen M, Smith JR, Sinha AA (2012) Global transcriptional analysis of psoriatic skin and blood confirms known diseaseassociated pathways and highlights novel genomic "hot spots" for differentially expressed genes. Genomics 100: 18-26. https://doi. org/10.1016/j.ygeno.2012.05.004

Dowlatshahi EA, van der Voort EA, Arends LR, Nijsten T (2013) Markers of systemic inflammation in psoriasis: a systematic review and meta-analysis. $\mathrm{Br} J$ Dermatol 169: 266-282. https://doi. org/10.1111/bjd.12355

Emmerson E, Campbell L, Ashcroft GS, Hardman MJ (2010) The phytoestrogen genistein promotes wound healing by multiple independent mechanisms. Mol Cell Endocrinol 321: 184-193. https://doi. org/10.1016/j.mce.2010.02.026

Galluzzo M, Talamonti M, De Simone C, D'Adamio S, Moretta G, Tambone S, Caldarola G, Fargnoli MC, Peris K, Bianchi L (2018) Secukinumab in moderate-to-severe plaque psoriasis: a multi-center, retrospective, real-life study up to 52 weeks observation. Expert Opin Biol Ther 18: 727-735. https://doi.org/10.1080/14712598.201 8.1481503

González R, Ballester I, López-Posadas R, Suárez MD, Zarzuelo A, Martínez-Augustin O, Sánchez de Medina F (2011) Effects of flavonoids and other polyphenols on inflammation. Crit Rev Food Sci Nutr 51: 331-362. https://doi.org/10.1080/10408390903584094

Gudjonsson JE, Ding J, Johnston A, Tejasvi T, Guzman AM, Nair RP, Voorhees JJ, Abecasis GR, Elder JT (2010) Assessment of the psoriatic transcriptome in a large sample: additional regulated genes and comparisons with in vitro models. J Invest Dermatol 130: 1829-1840. https://doi.org/10.1038/jid.2010.36

Gudjonsson JE, Ding J, Li X, Nair RP, Tejasvi T, Qin ZS, Ghosh D, Aphale A, Gumucio DL, Voorhees JJ, Abecasis GR, Elder JT
(2009) Global gene expression analysis reveals evidence for decreased lipid biosynthesis and increased innate immunity in uninvolved psoriatic skin. J Invest Dermatol 129: 2795-2804. https://doi. org/10.1038/jid.2009.173

Gupta SC, Tyagi AK, Deshmukh-Taskar P, Hinojosa M, Prasad S, Aggarwal BB (2014) Downregulation of tumor necrosis factor and other proinflammatory biomarkers by polyphenols. Arch Biochem Biophys 559: 91-99. https://doi.org/10.1016/j.abb.2014.06.006

Hsu L, Snodgrass BT, Armstrong AW (2014) Antidrug antibodies in psoriasis: a systematic review. Br J Dermatol 170: 261-273. https:// doi.org/10.1111/bjd.12654.

Ito K, Namikawa S, Takeuchi K (1992) Effect of the dry distillation tar of delipidated soybean (Glyteer) on a psoriasic model in the mouse (4). Nihon Yakurigaku Zasshi 99: 55-62. doi:10.1254/fpj.99.55

Korkina L, De Luca C, Pastore S (2012) Plant polyphenols and human skin: friends or foes. Ann N Y Acad Sci 1259: 77-86. https://doi. org/10.1111/j.1749-6632.2012.06510.x

Lee YK, Park OJ (2013) Soybean isoflavone genistein regulates apoptosis through $\mathrm{NF}-x \mathrm{~B}$ dependent and independent pathways. Exp Toxicol Pathol 65: 1-6. https://doi.org/10.1016/j.etp.2011.05.001

Martin DA, Towne JE, Kricorian G, Klekotka P, Gudjonsson JE, Krueger JG, Russell CB (2013) The emerging role of IL-17 in the pathogenesis of psoriasis: preclinical and clinical findings. I Invest Dermatol 133: 17-26. https://doi.org/10.1038/jid.2012.194

Mirahmadi SM, Shahmohammadi A, Rousta AM, Azadi MR, Fahanik-Babaei J, Baluchnejadmojarad T, Roghani M (2018) Soy isoflavone genistein attenuates lipopolysaccharide-induced cognitive impairments in the rat via exerting anti-oxidative and anti-inflammatory effects. Cytokine 104: 151-159. https://doi.org/10.1016/j. cyto.2017.10.008

Molyneux RJ, Lee ST, Gardner DR, Panter KE, James LF (2007) Phytochemicals: The good, the bad and the ugly? Phytochemistry 68: 2973-2985

Nakamura Y. Miyoshi N (2010) Electrophiles in foods: The current status of isothiocyanates and their chemical biology. Biosci Biotechnol Biochem 74: 242-255

Nijveldt RJ, van Nood E, van Hoorn DE, Boelens PG, van Norren K, van Leeuwen PA (2001) Flavonoids: A review of probable mechanisms of action and potential applications. Am J Clin Nutr 74: 418-425

Pazyar N, Yaghoobi R (2015) Soybean: a potential antipsoriasis agent. Jundishapur J Nat Pharm Prod 10: e20924. https://doi.org/10.17795/ jjnpp-20924

Shyong EQ, Lu Y, Lazinsky A, Saladi RN, Phelps RG, Austin LM, Lebwohl M, Wei H (2002) Effects of the isoflavone 4',5,7-trihydroxyisoflavone (genistein) on psoralen plus ultraviolet A radiation (PUVA)-induced photodamage. Carcinogenesis 23: 317-321. https:// doi.org/10.1093/ $\operatorname{carcin} / 23.2 .317$

Smolińska E, Moskot M, Jakóbkiewicz-Banecka J, Węrzynn G, Banecki B, Szczerkowska-Dobosz A, Purzycka-Bohdan D, Gabig-Cimińska M (2018) Molecular action of isoflavone genistein in the human epithelial cell line HaCaT. PLoS ONE 13: e0192297. https://doi. org/10.1371/journal.pone.0192297

Tian S, Krueger JG, Li K, Jabbari A, Brodmerkel C, Lowes MA, Suárez-Fariñas M, (2012) Meta-analysis derived (MAD) transcriptome of psoriasis defines the "core" pathogenesis of disease. PLoS One 7: e44274. https://doi.org/10.1371/journal.pone.0044274

Vivatvakin S, Werawatganon D, Somanawat K, Klaikeaw N, Siriviriyakul P (2017) Genistein-attenuated gastric injury on indomethacininduced gastropathy in rats. Pharmacogn Mag 13 (Suppl 2): S306S310. https://doi.org/10.4103/pm.pm_502_16. 